\title{
Type 3 fimbriae, encoded by the conjugative plasmid pOLA52, enhance biofilm formation and transfer frequencies in Enterobacteriaceae strains
}

\section{Correspondence \\ Lars Hestbjerg Hansen hestbjerg@bi.ku.dk}

Received 7 July 2007

Revised 30 August 2007

Accepted 3 September 2007

\author{
Mette Burmølle, ${ }^{1}$ Martin lain Bahl, ${ }^{1}$ Lars Bogø Jensen, ${ }^{2}$ \\ Søren J. Sørensen ${ }^{1}$ and Lars Hestbjerg Hansen ${ }^{1}$ \\ ${ }^{1}$ Department of Microbiology, University of Copenhagen, 1307 Copenhagen K, Denmark \\ ${ }^{2}$ Unit for Antimicrobial Resistance, The National Food Institute, DTU, Denmark
}

\begin{abstract}
The conjugative plasmid pOLA52, which confers resistance to olaquindox and other antimicrobial agents through a multidrug efflux pump, was investigated for its ability to promote biofilm formation in Escherichia coli. Screening of a transposon-mutagenized pOLA52 clone library revealed several biofilm-deficient mutants, which all mapped within a putative operon with high homology to the mrkABCDF operon of Klebsiella pneumoniae, where these genes are responsible for type 3 fimbriae expression, attachment to surfaces and biofilm formation. Biofilm formation in microtitre plates and in urinary catheters of clones containing pOLA52 with a disrupted putative mrk operon was reduced by more than 100-fold and 2-fold, respectively, compared to mutants with an intact mrk operon. The conjugative transfer rate of pOLA52 was also significantly lower when the mrk operon was disrupted. Through reverse transcriptase analysis, it was demonstrated that the genes contained in the putative mrk operon were linked and likely to be expressed as a single operon. Immunoblotting with type 3 fimbriae (MrkA)-specific antibodies further verified expression of type 3 fimbriae. When transferred to other, potentially pathogenic, members of the family Enterobacteriaceae, including Klebsiella pneumoniae, Salmonella Typhimurium, Kluyvera sp. and Enterobacter aerogenes, pOLA52 facilitated increased biofilm formation. pOLA52 is believed to represent the first example of a conjugative plasmid encoding type 3 fimbriae, resulting in enhanced conjugation frequencies and biofilm formation of the plasmid-harbouring strain.
\end{abstract}

\section{INTRODUCTION}

The plasmid pOLA52 was exogenously isolated from swine manure and demonstrated to confer resistance to the antimicrobial agent olaquindox (Sørensen et al., 2003). This compound was extensively used in pig farming as a growth promoter in the European Union until 1999 and is still in use in other countries. The resistance mechanism of pOLA52 was found to depend on a resistance-nodulationcell-division (RND) family efflux system (Hansen et al., 2004). Apart from olaquindox, the pump exports chloramphenicol and ethidium bromide across the membrane of Escherichia coli cells (Hansen et al., 2004) and recently experiments have demonstrated reduced susceptibility to other antibiotics, detergents and disinfectants (Hansen et al., 2007). Independent of the efflux pump system, pOLA52 confers resistance to the $\beta$-lactam antibiotic ampicillin (Hansen et al., 2004, 2007).

Abbreviations: CV, crystal violet; RND, resistance-nodulation-cell division.
Plasmid pOLA52 was recently sequenced (to be published elsewhere; A. Norman, Q. She, S. J. Sørensen \& L. H. Hansen) and annotation revealed a putative operon, totalling $5.6 \mathrm{~kb}$, consisting of five genes highly homologous to the mrkABCDF genes contained in the mrk operon of Klebsiella pneumoniae (Allen et al., 1991). In K. pneumoniae, the mrk operon encodes type 3 fimbriae, which are involved in attachment to and biofilm formation on biotic and abiotic surfaces, including different human cell types (Hornick et al., 1991; Livrelli et al., 1996; Schurtz et al., 1994; Tarkkanen et al., 1997). The $m r k A$ and $m r k D$ genes encode the major structural component of the type 3 fimbriae and an adhesin, respectively (Allen et al., 1991; Jagnow \& Clegg, 2003; Langstraat et al., 2001). The genes $m r k B$ and $m r k C$, which encode proteins essential for fimbrial expression, are thought to be part of the fimbrial assembly process via the chaperone-usher pathway (Allen et al., 1991; Gerlach et al., 1988; Sauer et al., 2000). The $m r k F$ gene encodes the MrkF protein, which is thought to be involved in stabilizing intact fimbriae (Allen et al., 1991). 
K. pneumoniae is an opportunistic pathogen, which is often associated with infections in the human respiratory and urinary tract. Because cell attachment is an essential step in infection, the type 3 fimbriae may be crucial for $K$. pneumoniae virulence (Livrelli et al., 1996; Williams \& Tomas, 1990). In several other members of the family Enterobacteriaceae, adhesion-mediating fimbriae and pili are believed to be essential for virulence (Hornick et al., 1991). The mrkABCDF genes of pOLA52 are the first type 3 fimbriae-encoding genes reported to be present on a plasmid. Previously, plasmid-borne fimbrial genes, different from the mrk genes, have been reported to be involved in aggregative adherence of $E$. coli to mucosal cells, causing traveller's diarrhoea and persistent diarrhoea among infants, especially in the developing world (Levine, 1987; Vila et al., 2000), and also to contribute to the pathogenicity of E. coli O157 (Brunder et al., 2001).

Biofilms are recognized as surface-attached bacteria embedded in a self-produced matrix, composed mainly of polysaccharides, but also containing proteins and nucleic acids (Sutherland, 2001). Factors important for biofilm formation include production of extracellular polymers and cell surface-attached structures including flagella, fimbriae and curli (Castonguay et al., 2006; Klausen et al., 2006). Pili involved in conjugation of Gram-negative bacteria have also been shown to promote biofilm formation in several studies (Dudley et al., 2006; Ghigo, 2001; Reisner et al., 2003, 2006). Ghigo (2001) demonstrated how biofilm formation of an $E$. coli strain was dependent on whether this strain contained a conjugative plasmid and expressed the conjugative pili. Dudley et al. (2006) recently demonstrated that thin type IV pili, different from the thicker conjugative pili, but also involved in conjugation, promoted cell-cell attachment as well as attachment to abiotic surfaces and thereby promoted biofilm formation.

Bacteria organized in biofilms cause many human infections. They attach to human cell tissue, to teeth, and to medical devices such as prosthetic heart valves and urinary catheters (Donlan \& Costerton, 2002). Biofilms on urinary catheters often involve Enterobacteriaceae such as E. coli, K. pneumoniae and Enterobacter aerogenes (Donlan \& Costerton, 2002). These biofilm-associated infections are hard to cure due to an increased resistance of the biofilm bacteria to antibiotics and phagocytosis by inflammatory cells, and thus represent a large medical problem (Donlan \& Costerton, 2002; Fux et al., 2005; Jefferson, 2004). Because of the increased protection of bacteria in biofilm, they should achieve a higher fitness in this life form compared to the free-living, planktonic state (Jefferson, 2004). A selective pressure may therefore exist, favouring the prevalence of genes encoding biofilm-associated traits, like plasmid-encoded pili or fimbriae.

In our laboratory we have observed an increased biofilm phenotype in an E. coli CSH26 strain harbouring plasmid pOLA52. The aim of this study was to investigate whether the putative type 3 fimbriae-encoding operon, with homology to the mrk operon from K. pneumoniae, is expressed and could be responsible for the biofilm phenotype.

\section{METHODS}

Strains and plasmids used in this study. The strains and plasmids used in this study are listed in Table 1.

Media and culture conditions. Bacteria were grown in LuriaBertani (LB) medium (Sambrook et al., 1989). Solid medium contained $15 \mathrm{~g}$ agar $1^{-1}$. Bacterial cultures were incubated at $37{ }^{\circ} \mathrm{C}$; LB broths were incubated on a rotary shaker at 250 r.p.m. Phosphatebuffered saline (PBS) was used for washing cells and preparing serial dilutions (Sambrook et al., 1989). The antibiotics kanamycin (Kan, $50 \mu \mathrm{g} \mathrm{ml}^{-1}$ ), ampicillin (Amp, $100 \mu \mathrm{g} \mathrm{ml}^{-1}$ ), tetracycline (Tet, $20 \mu \mathrm{g} \mathrm{m} \mathrm{m}^{-1}$ ) and rifampicin (Rif, $100 \mu \mathrm{g} \mathrm{ml} \mathrm{m}^{-1}$ ) were used when required.

\begin{abstract}
Construction of a clone library with $a \operatorname{Kan}^{\mathbf{R}}$ entranceposon randomly inserted in pOLA52. A knockout library of pOLA52 was created. First, pENTRANCEPOSON $\left(\operatorname{Kan}^{\mathrm{R}}\right)$ (Finnzymes) was digested with BglII restriction endonuclease. The entranceposon was then excised from an agarose gel as a $1.2 \mathrm{~kb}$ fragment and purified using the QIAEX II Gel Extraction kit (Qiagen). Two micrograms of a plasmid preparation of pOLA52 (Plasmid Mini AX kit, A\&A Biotechnology) was incubated with $20 \mathrm{ng}$ entranceposon and $1 \mu \mathrm{l}$ MuA transposase in MuA transposase buffer according to the manufacturer's protocol (Finnzymes). The transposition reaction was diluted 10-fold in sterile deionized water, electroporated into $E$. coli Genehogs (Invitrogen), and successful transpositions of the entranceposon into pOLA52 were selected for on solid LB medium containing Kan. Three hundred transformants, E. coli Genehogs/ pOLA52::Kan ${ }^{\mathrm{R}}$, each representing a different, randomly distributed transposition event in pOLA52, were then frozen in microtitre plates in $17 \%$ glycerol at $-80{ }^{\circ} \mathrm{C}$ for later use.
\end{abstract}

Screening of the clone library for the ability to form biofilm. A total of 88 of the transformants described above were randomly selected and screened for their ability to form biofilm in microtitre plate wells, by use of a previously established method in which cell attachment and biofilm formation are quantified by staining with crystal violet (CV) (O'Toole \& Kolter, 1998). E. coli CSH26 with and without pOLA52 were included as reference strains. The 88 clones were inoculated from glycerol stocks directly into microtitre plates containing $200 \mu \mathrm{LB}$ broth and grown with agitation (200 r.p.m.) for $24 \mathrm{~h}$. Staining and quantification of attached cells were performed as previously described (Burmølle et al., 2006), using an EL 340 microplate reader (Bio-Tek Instruments) for absorbance measurements at $595 \mathrm{~nm}$.

Of the 88 clones, 7 were biofilm negative. The biofilm-forming ability of these clones and that of 12 randomly selected biofilm-positive clones was verified by performing the assay in four replicates for each strain. Equal densities $\left(\mathrm{OD}_{600} \pm 10 \%\right)$ of the bacteria were diluted 10 -fold in LB in microtitre plate wells to a total volume of $200 \mu$ l. The plates were incubated as described above. Wells containing only LB were used as negative controls and the absorbance measurements of these wells were subtracted from the experimental measurements.

Sequencing of biofilm-negative and -positive clones. Of the 19 clones tested above, the 7 biofilm-negative and 4 randomly selected, biofilm-positive clones were chosen for sequencing to identify the exact location of the entranceposon in the pOLA52 derivatives as 
Table 1. Strains and plasmids

\begin{tabular}{|c|c|c|}
\hline Strain or plasmid & Relevant characteristics & Reference \\
\hline \multicolumn{3}{|l|}{ Strains } \\
\hline Escherichia coli Genehogs & & Invitrogen \\
\hline Escherichia coli Genehogs ${ }^{\text {Rif }}$ & Spontaneous Rif ${ }^{\mathrm{R}}$ mutant & This study \\
\hline Escherichia coli $\mathrm{CSH} 26$ & $\begin{array}{l}\text { Requires proline and leucine for growth } \\
\text { (ara } \Delta(\text { lac-pro }) \text { thi })\end{array}$ & Miller (1972) \\
\hline Escherichia coli MC4100 & Prototroph & Silhavy et al. (1984) \\
\hline Salmonella Typhimurium DT27 & & Olsen et al. (2004) \\
\hline Klebsiella pneumoniae DSA712 & & Dorthe Sandvang* \\
\hline Kluyvera sp. MB101 & & Burmølle et al. (2005) \\
\hline Enterobacter aerogenes DSM30053 & & $\mathrm{DMSZ} \dagger$ \\
\hline \multicolumn{3}{|l|}{ Plasmids } \\
\hline pOLA52 & Biofilm positive & Sørensen et al. (2003) \\
\hline pOLA52-bla:: $\operatorname{Kan}^{\mathrm{R}}$ & Biofilm positive, $\mathrm{Amp}^{\mathrm{S}} \mathrm{Kan}^{\mathrm{R}}$ & This study \\
\hline pOLA52-oqxB:: $\operatorname{Kan}^{\mathrm{R}}(\mathrm{H} 1)$ & Biofilm positive, $\operatorname{Kan}^{\mathrm{R}}$ & This study \\
\hline pOLA52-mrkC:: $\operatorname{Kan}^{\mathrm{R}}(\mathrm{H} 5)$ & Biofilm negative, $\mathrm{Kan}^{\mathrm{R}}$ & This study \\
\hline pLOW2 & Low-copy plasmid, $\mathrm{Kan}^{\mathrm{R}}$ & Hansen et al. (1997) \\
\hline pLOW2 : : $m r k A-F$ & Low-copy plasmid, $\operatorname{Kan}^{\mathrm{R}}$ & This study \\
\hline pLOW2 $::$ Tet $^{\mathrm{R}}-m r k A-F$ & Low-copy plasmid, $\operatorname{Kan}^{\mathrm{R}} \mathrm{Tet}^{\mathrm{R}}$ & This study \\
\hline
\end{tabular}

${ }^{\star}$ Dorthe Sandvang, Novozymes, Denmark.

$\dagger$ Deutsche Sammlung von Mikroorganismen und Zellkulturen GmbH.

illustrated in Fig. 1. pOLA52:: $\operatorname{Kan}^{\mathrm{R}}$ plasmids from the 11 chosen clones were purified as described above. From these purifications, $12 \mu \mathrm{l}$ plasmid DNA $(0.1-1.0 \mu \mathrm{g})$ was digested with NlaIII in reactions of $20 \mu \mathrm{l}$ at $37{ }^{\circ} \mathrm{C}$ for $60 \mathrm{~min}$, followed by heat inactivation of NlaIII at $65{ }^{\circ} \mathrm{C}$ for $25 \mathrm{~min}$. Then, $1 \mu \mathrm{l} \mathrm{T} 4$ ligase (10 units) and $2 \mu \mathrm{l} \mathrm{ATP}$ $(10 \mathrm{mM})$ was added and ligation was performed at $25{ }^{\circ} \mathrm{C}$ for $60 \mathrm{~min}$, followed by heat inactivation of T4 ligase at $65{ }^{\circ} \mathrm{C}$ for $25 \mathrm{~min}$. PCRs were performed with primers $\mathrm{Ent}(\mathrm{Kan}) \mathrm{FW}\left(5^{\prime}\right.$-TTATTCGGTCGAAAAGGATCCG- $3^{\prime}$ ) and Ent(Kan)Rev (5'-TCCCGTCAAGTCAGCGTA- $\left.3^{\prime}\right)$ and the PCR products were visualized by agarose gel electrophoresis. Sequencing reactions (DYEnamic ET dye terminator cycle sequencing kit, MegaBACE) containing $0.1 \mu \mathrm{g}$ purified PCR product and 10 pmol primer Ent(Kan)FW in total volumes of $10 \mu \mathrm{l}$ were conducted. Sequencing was performed using a MegaBACE 1000 sequencer (Molecular Dynamics). To locate the position of the $\operatorname{Kan}^{\mathrm{R}}$ cassette, the sequences obtained were compared to the plasmid sequence (A. Norman, Q. She, S. J. Sørensen \& L. H. Hansen, unpublished). Two clones were chosen for further analysis: the biofilm-positive E. coli Genehogs/pOLA52-oqxB:: $\operatorname{Kan}^{\mathrm{R}}(\mathrm{H} 1)$ and the biofilm-negative E. coli Genehogs/pOLA52-mrkC:: $\operatorname{Kan}^{\mathrm{R}}(\mathrm{H} 5)$; see Table 1 .

Complementation of biofilm formation. To investigate whether the biofilm formation was solely caused by the expression of the mrk operon, the absence of the biofilm phenotype was complemented in E. coli Genehogs and in strain $\mathrm{H} 5$ containing mrkC-mutagenized
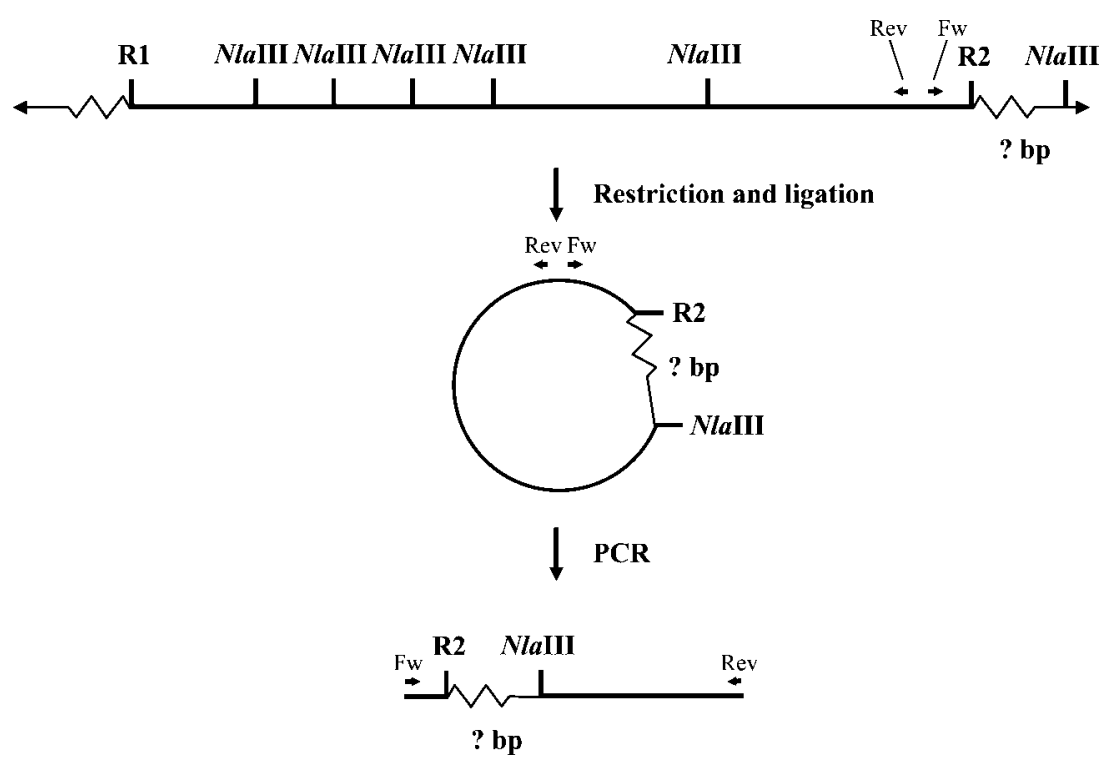

Fig. 1. The $\operatorname{Kan}^{\mathrm{R}}$ cassette contains several Nlalll recognition sites; therefore Nlalll digestion of the $\mathrm{Kan}^{\mathrm{R}}$ mutagenized plasmid will, when the products are ligated, result in many different circular fragments. Some of these fragments contain part of the $\mathrm{Kan}^{\mathrm{R}}$ cassette and part of the original plasmid, and they will also contain the primer annealing sites. PCRs will therefore result in amplification of these circular fragments, and by sequencing of the PCR products, the location of the $\mathrm{Kan}^{\mathrm{R}}$ insertion can be determined. 
pOLA52. Two primers, mrkFW (5'-AAGTGCACGGGGGAGACTTACCCGAAATCT- $3^{\prime}$ ) and mrkRev (5'-GCAGCGGCCGCATTCCCGGCTTTTTAAC-3'), were designed to amplify the entire $m r k A-F$ operon as a $6680 \mathrm{bp}$ fragment with an ApaLI site at one end and a NotI site at the other (restriction sites are underlined). Following double digestion of both PCR product and vector with ApaLI and NotI, the mrk operon was directionally cloned into pLOW2 (Hansen et al., 1997) and transformed into E. coli Genehogs. The kanamycin resistance of pLOW2 was knocked out by an Entranceposon $\left(\mathrm{Tet}^{\mathrm{R}}\right.$ ) insertion into the $a p h\left(3^{\prime}\right)$ gene of the resulting pLOW2-mrkA-F plasmid as described above and according to the protocol of the manufacturer (Finnzymes, Finland). This resulted in a selective marker, tetracycline resistance, different from the kanamycin resistance on the pOLA52 derivative in $\mathrm{H} 5$. The position of the Entranceposon $\left(\mathrm{Tet}^{\mathrm{R}}\right)$ in the aph $\left(3^{\prime}\right)$ gene was verified by the phenotypic loss of kanamycin resistance and by sequencing. Then the H5 strain as well as E. coli Genehogs without the plasmid were transformed with the Tet ${ }^{\mathrm{R}}$ pLOW2:: Tet $^{\mathrm{R}}-m r k A-F$ plasmid and subjected to the microtitre-biofilm assay described above.

Biofilm formation on urinary catheters. E. coli Genehogs/ pOLA52-oqxB:: $\operatorname{Kan}^{\mathrm{R}}$ (H1) and E. coli Genehogs/pOLA52$m r k C:: \operatorname{Kan}^{\mathrm{R}}$ (H5) were tested for their ability to form biofilms on medical urinary catheters. The strains were grown overnight in LB broth (containing Kan), and the cell density was adjusted $\left(\mathrm{OD}_{600}\right.$ $\pm 10 \%)$. From these cultures, $50 \mu \mathrm{l}$ was transferred to glass tubes containing $5 \mathrm{ml} \mathrm{LB}$ and three pieces of urinary catheter tubing (AquaFlate Glycerine Silicone, Ref. 178305; Rüsch, Kernen, Germany), cut vertically to eliminate channels without bacterial movement and horizontally to obtain $1 \mathrm{~cm}$ pieces. Catheter tubing in LB, but not exposed to bacteria, was used as negative control and the experiment was performed in triplicate. The tubes were incubated with gentle shaking for $24 \mathrm{~h}$ at $37^{\circ} \mathrm{C}$. The cell suspension was then discarded and the catheter tubing pieces were rinsed twice with $10 \mathrm{ml}$ PBS, stained for $20 \mathrm{~min}$ in CV (1\%) and washed twice with PBS. Two millilitres of ethanol was added to dissolve the CV bound to the attached cells and $A_{595}$ was measured. The absorbance value of the negative control was subtracted from the measurements.

Exposure to inhibitory compounds. An $\mathrm{Amp}^{\mathrm{S}}$ clone from the $E$. coli Genehogs/pOLA52-bla:: $\operatorname{Kan}^{\mathrm{R}}$ library was isolated as previously described (Hansen et al., 2007) . Overnight cultures of this strain (E. coli Genehogs/pOLA52-bla::Kan $\left.{ }^{\mathrm{R}}\right)$, E. coli Genehogs/pOLA52oqxB:: $\operatorname{Kan}^{\mathrm{R}}(\mathrm{H} 1)$ and E. coli Genehogs/pOLA52-mrkC:: $\operatorname{Kan}^{\mathrm{R}}(\mathrm{H} 5)$ were diluted 100 -fold in microtitre plate wells containing LB medium. The inhibitory compounds tetracycline (Tet) and hydrogen peroxide $\left(\mathrm{H}_{2} \mathrm{O}_{2}\right)$ were added to obtain the following final concentrations: Tet, $0.5,2,10$ and $20 \mu \mathrm{g} \mathrm{ml}^{-1} ; \mathrm{H}_{2} \mathrm{O}_{2}, 35,175,875$ and $1750 \mu \mathrm{g} \mathrm{ml} l^{-1}$. Wells without inhibitory compounds were included, and wells containing only LB served as background. The total volume of each well was $200 \mu \mathrm{l}$. Each treatment was performed in triplicate wells. The plate was incubated with shaking (200 r.p.m.) at $37{ }^{\circ} \mathrm{C}$. After $1 \mathrm{~h}$, the respiratory indicator 2,3,5-triphenyltetrazolium chloride (TTC) was added to each well at a final concentration of $0.01 \%$, and the $A_{490}$ was measured after $48 \mathrm{~h}$ in an EL 340 microplate reader (Bio-Tek Instruments). The activity in wells exposed to inhibitory compounds was then related to that of the equivalent strain without exposure to inhibitory compounds.

RT-PCR analysis of the putative mrk operon. Reverse transcription-PCR (RT-PCR) was performed to verify transcription of the putative mrk operon and the linkage of the genes contained in the operon at the mRNA level. Due to the length of the putative mrk operon, the RT reactions were performed by use of three reverse primers, annealing at various positions on the operon, followed by nested PCRs to increase the specificity. The PCR primers were designed to demonstrate the linkage of the genes $m r k A-m r k B$, $m r k B-m r k C, m r k C-m r k D$ and $m r k D-m r k F$, respectively. Total RNA was purified from $1.5 \mathrm{ml}$ of overnight culture of $E$. coli Genehogs (no plasmid) and E. coli Genehogs/pOLA52-oqxB:: $\operatorname{Kan}^{\mathrm{R}}$ (H1) (Genelute Bacterial Total RNA Purification kit, Sigma). The purified RNA was then subjected to DNase digestion and subsequent DNase inhibition, by use of a DNA-free kit (Ambion) as recommended. This RNA was used as template in RT (Expand Reverse Transcriptase kit, Roche). A total of $8 \mu \mathrm{g}$ was used in a total of six (three for each strain) $20 \mu \mathrm{l}$ RT reactions $\left(65{ }^{\circ} \mathrm{C}\right.$ for $10 \mathrm{~min}$, subsequent cooling on ice, followed by $43{ }^{\circ} \mathrm{C}$ for $\left.60 \mathrm{~min}\right)$ containing $20 \mathrm{pmol}$ of the primers mrkFrevl $\left(5^{\prime}\right.$ ATATTCTGCGCTCCATCC- $3^{\prime}$ ), mrkCrev (5'-CGCTCTGACGATAGCCTT-3') or mrkBrev (5'-CATCGGCGCCATAGTCAT-3'). Next, four nested PCR reactions (Phusion, Finnzymes) were performed, using $1 \mu \mathrm{l}$ of RT product (10-fold diluted in $\mathrm{H}_{2} \mathrm{O}$ ) as template, as follows. The RT product obtained from the reverse primer mrkBrev was used as template in a nested PCR performed with the primers mrkBrevnst1 (5'-GCGGGGTGATAATAAACG$\left.3^{\prime}\right)+$ mrkAfw $\left(5^{\prime}\right.$-GCGGCCAGGTTAATTTCT- $\left.3^{\prime}\right)$. The mrkCrevRT product was used as template in a nested PCR with primers mrkCrevnst 1 (5'-ACCAGGTTGTCTTTGACG-3') $3^{\prime}$ mrkBfw $\quad\left(5^{\prime}-\right.$ GCAGTGAAAGTGGCCGTT- ${ }^{\prime}$ ), and the RT product obtained from mrkFrev1 served as template in two nested PCRs with the primer sets mrkFrev2 (5'-ACCGCGATCGTGGACTTCC-3') + mrkDfw (5' -TTAGCACCTCGTTCTCCG-3'), and mrkDrev (5'-CGGAGAACGAGGTGCTAA-3 $\left.{ }^{\prime}\right)+$ mrkCfw (5'-AAGGCTATCGTCAGAGCG-3'). Control nested PCRs, similar to those described above, were performed using the DNase-treated RNA (with twice the RNA concentration used in the RT-PCR reactions) as template to verify that the RNA was DNA-free.

Immunoblotting with MrkA-specific antibodies. A colony immunoblot was performed to verify that the expression of the putative mrk operon produced intact type 3 fimbriae. Rabbit antiserum raised against the $K$. pneumoniae type 3 fimbriae major subunit MrkA (Di Martino et al., 2003) was provided as a gift from Arlette Darfeuille-Michaud, Pathogénie Bactérienne Intestinale, USCINRA 2018 CBRV, Clermont Ferrand, France. From overnight LB broth cultures of E. coli Genehogs (no plasmid), E. coli Genehogs/ pOLA52-oqxB:: $\operatorname{Kan}^{\mathrm{R}}(\mathrm{H} 1)$ and $K$. pneumoniae DSA712, $5 \mu$ l was spotted onto a nitrocellulose filter placed on an LB agar plate, grown for $16 \mathrm{~h}$ and dried at $80^{\circ} \mathrm{C}$ for $2 \mathrm{~h}$. Then the membrane was blocked in $50 \mathrm{ml} 5 \%$ skim milk (Difco) for $1 \mathrm{~h}$ on a slowly rotating platform shaker at room temperature. The rabbit antiserum was added at a $1: 500$ dilution in $5 \%$ skim milk solution and incubated for $1.5 \mathrm{~h}$ on the platform shaker at room temperature, after which the membrane was washed three times for $10 \mathrm{~min}$ in TBS buffer $(125 \mathrm{mM} \mathrm{NaCl}$,

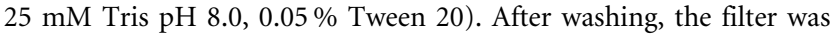
incubated for $1 \mathrm{~h}$ at room temperature with peroxidase-labelled goat anti-rabbit immunoglobulin G (DAKO) in TBS at 2000-fold dilution. The membrane was washed again three times for $10 \mathrm{~min}$ in TBS buffer and resuspended in $50 \mathrm{ml}$ fresh TBS buffer. For the detection of expressed MrkA protein, $20 \mathrm{mg}$ 4-chloro-1-naphthol (Sigma) and $0.2 \% \mathrm{H}_{2} \mathrm{O}_{2}$ were added and the membrane was allowed to develop for $30 \mathrm{~min}$.

Conjugation frequency. Plasmids pOLA52-oqxB:: $\mathrm{Kan}^{\mathrm{R}}$ and

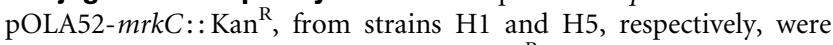
transferred separately to spontaneous $\mathrm{Rif}^{\mathrm{R}}$ E. coli Genehogs cells $\left(\mathrm{H} 1^{\mathrm{Rif}}\right.$ and $\left.\mathrm{H}^{\mathrm{Rif}}\right)$ by conjugation and these transconjugants were used as donor cells in the following conjugation experiment. Single colonies of the donor strains were transferred to separate tubes containing $5 \mathrm{ml} \mathrm{LB}$ broth supplemented with Rif and Kan and incubated to an $\mathrm{OD}_{600}$ of 0.4 , after which the cultures were washed twice in LB broth $(5000 \mathrm{~g}, 5 \mathrm{~min})$ and the $\mathrm{OD}_{600}$ was adjusted to 0.2. The recipient E. coli MC4100 strain was prepared analogously; however, this strain was grown in the absence of antibiotics and the wash-steps were omitted. Triplicate transfer experiments were 
conducted by mixing $40 \mu \mathrm{l}$ of either the $\mathrm{H} 1^{\text {Rif }}$ or $\mathrm{H} 5^{\text {Rif }}$ donor strain with $160 \mu \mathrm{l}$ of the recipient strain in micro-centrifuge tubes, incubated at $37^{\circ} \mathrm{C}$ overnight without shaking. Serial dilutions of the mating mixtures were prepared and the following solid media were used for selective plating: LB-agar containing Rif + Kan, minimal media plates (de Lipthay et al., 2001) containing glucose and minimal media plates containing glucose and Kan for selection of donors, recipients + transconjugants and transconjugants respectively.

Biofilm formation of potentially pathogenic Enterobacteriaceae strains \pm pOLA52. The plasmid (pOLA52-bla::Kan ${ }^{\mathrm{R}}$ ) from the $\mathrm{Amp}^{\mathrm{S}}$ clone, isolated above, was purified and transferred to $E$. coli $\mathrm{CSH} 26$ by electroporation, followed by a conjugative transfer to Salmonella Typhimurium DT27, Klebsiella pneumoniae DSA712, Kluyvera sp. MB101 and Enterobacter aerogenes DSM30053, as previously described (Hansen et al., 2007). These strains, with and without pOLA52, were grown overnight in $\mathrm{LB} \pm \mathrm{Kn}$; $\mathrm{OD}_{600}$ was measured, and the cultures were diluted in LB to assure similar cell densities of each strain pair (identical species \pm plasmid). E. coli $\mathrm{CSH} 26 \pm$ pOLA52 was used as control. Then, $20 \mu \mathrm{l}$ samples were transferred to microtitre plate wells, containing $180 \mu \mathrm{LB}$, in four well replicates. The plate was incubated, and the attached cells were stained and quantified as described above. The biofilm formation of each strain devoid of plasmid was then related to that of its plasmidcontaining counterpart.

\section{RESULTS AND DISCUSSION}

The sequencing of pOLA52 revealed a gene cluster with high homology to the chromosomal mrk operon of $K$. pneumoniae, which encodes type 3 fimbriae (Allen et al., 1991). In K. pneumoniae, expression of type 3 fimbriae is essential for attachment to abiotic and biotic surfaces and for biofilm formation (Di Martino et al., 2003; Jagnow \& Clegg, 2003; Langstraat et al., 2001; Tarkkanen et al., 1997). The latter includes attachment of K. pneumoniae to human epithelial and urinary bladder cells, which may be involved in the common human infections caused by K. pneumoniae (Tarkkanen et al., 1997).

The complete mrk operon of K. pneumoniae contains six genes, of which five homologues are present in pOLA52 (Allen et al., 1991). The remaining gene, $m r k E$, has been suggested to encode a product involved in regulation of expression of type 3 fimbriae; however, strains lacking the $m r k E$ gene expressed type 3 fimbriae at levels similar to the wild-type, which suggests that MrkE is not essential for fimbriae expression (Allen et al., 1991). The five translated open reading frames showed an $81-95 \%$ identical amino acid sequence when compared to the mrk operon from $K$. pneumoniae (A. Norman, Q. She, S. J. Sørensen \& L. H. Hansen, unpublished).

\section{Construction of clone library and screening for loss of biofilm formation phenotype}

Due to the close homology between the pOLA52 gene cluster and the K. pneumoniae mrk operon, we hypothesized that pOLA52 would promote biofilm formation, and this was confirmed by preliminary screenings for biofilm formation by $E$. coli CSH26 \pm pOLA52. In order to evaluate what specific genes or operons of pOLA52 contributed to biofilm formation when expressed by E. coli Genehogs, 88 clones containing pOLA52 with random $\mathrm{Kan}^{\mathrm{R}}$ insertions were screened for their ability to form biofilm. Of the $88 \mathrm{E}$. coli Genehogs/pOLA52:: Kan $^{\mathrm{R}}$ transformants, 7 appeared to be deficient in biofilm formation. When tested in quadruplicate, on average an 80-fold reduction in biofilm formation was observed in these 7 strains when compared to the 4 biofilm-forming clones selected for sequencing, described later (Fig. 2). Thus, specific genes encoded by pOLA52 induced biofilm formation.

Plasmids were subjected to NlaIII digestion, T4 ligation and PCR as illustrated in Fig. 1. The insertion of the entranceposon was localized to the putative mrk operon of pOLA52 in all of the seven biofilm-negative E. coli Genehogs/pOLA52:: $\operatorname{Kan}^{\mathrm{R}}$ transformants by sequencing (Fig. 3). In each of the four biofilm-positive transformants, the insertion mapped in genes encoding proteins with known functions (topB, toxB, soj and $o q x B$ ), outside the putative mrk operon. This strongly indicates that the putative mrk operon, homologous to that encoding type 3 fimbriae in K. pneumoniae (Allen et al., 1991), is essential for the biofilm-inducing capability of pOLA52.

\section{Biofilm formation on abiotic surfaces}

Two clones, E. coli Genehogs/pOLA52-oqxB:: $\operatorname{Kan}^{\mathrm{R}}(\mathrm{H} 1)$ with the transposon inserted in the $o q \times B$ gene, encoding the efflux pump, and E. coli Genehogs/pOLA52$m r k C:: \operatorname{Kan}^{\mathrm{R}}$ (H5) with the transposon inserted in the $m r k C$ gene homologue, were chosen as representatives of biofilm and non-biofilm phenotypes, respectively. When the two clones were grown in microtitre plates and stained with $\mathrm{CV}$, there was more than a 100 -fold, statistically

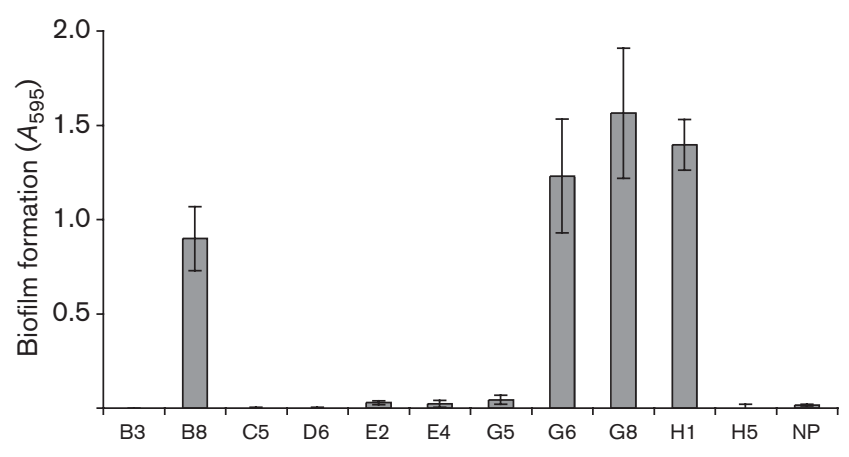

Fig. 2. Biofilm formation of seven biofilm-negative (B3, C5, D6, $\mathrm{E} 2, \mathrm{E} 4, \mathrm{G} 5, \mathrm{H} 5)$ and four (randomly picked) biofilm-positive (B8, G6, G8, H1) E. coli Genehogs/pOLA52:: $\operatorname{Kan}^{\mathrm{R}}$ clones. E. coli Genehogs without plasmid pOLA52 (NP) was included as a negative control. The cells were incubated in microtitre plate wells for $24 \mathrm{~h}$, followed by quantification of biofilm formation by staining of attached cells and matrix with CV and spectrophotometric absorbance measurements $\left(A_{595}\right)$. Columns represent means $\pm \mathrm{SD}$ for four replicates. 


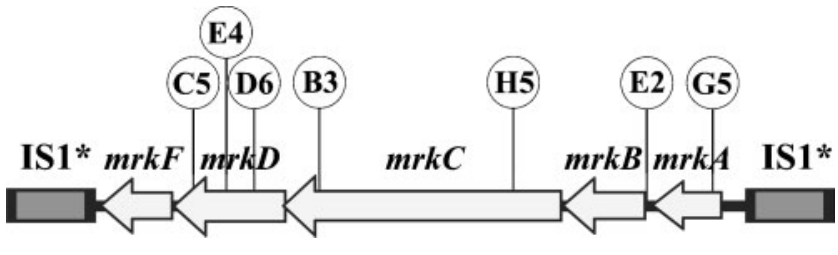

Fig. 3. Distribution of the $\mathrm{Kan}^{\mathrm{R}}$ insertion in the seven biofilmnegative clones (B3, C5, D6, E2, E4, G5, H5), all localizing in the putative $m r k$ operon. The $\mathrm{Kan}^{\mathrm{R}}$ insertions in the four biofilmpositive clones all localized outside this operon. The insertions were mapped by Nlall digestion of the mutagenized plasmid followed by ligation, PCR amplification and sequencing.

significant (Student's $t$-test, $P<0.01)$ difference in biofilm formation (Fig. 2).

The ability to form biofilm in microtitre plates could be fully complemented when E. coli Genehogs and strain H5 were transformed with plasmid pLOW2::Tet ${ }^{\mathrm{R}}-m r k A-F$. $\mathrm{CV}$ staining showed attachment to microtitre plates at levels slightly higher than those seen with the $\mathrm{H} 1$ derivative (data not shown), probably due to increased expression levels or copy number differences between the pLOW2 and the pOLA52 derivatives.

A twofold reduction (statistically significant, Student's $t$ test, $P<0.01)$ in biofilm formation was observed for $\mathrm{H} 5$, containing a disrupted putative $m r k$ operon, compared to that of $\mathrm{H} 1$, when the two clones were allowed to attach to silicone urinary catheters [biofilm formation $\left(A_{595}\right)=0.2$ and 0.09 for $\mathrm{H} 1$ and $\mathrm{H} 5$, respectively]. This implies that bacterial strains containing pOLA52 may have enhanced opportunities for establishment of biofilms on this surface and other medical devices.

Both E. coli Genehogs/pOLA52-bla:: $\operatorname{Kan}^{\mathrm{R}}$ and H1 were significantly more resistant than $\mathrm{H} 5$ to $0.5 \mu \mathrm{g}$ Tet $\mathrm{ml}^{-1}$ after $48 \mathrm{~h}$. Compared to the non-exposed equivalent strains, the activity of $\mathrm{H} 5$ (biofilm-negative) was reduced by $43.3 \%$, but by only $22.6 \%$ and $15.8 \%$ for $\mathrm{H} 1$ and E. coli Genehogs/pOLA52-bla:: $\operatorname{Kan}^{\mathrm{R}}$, respectively. The three higher concentrations of Tet resulted in near-total inhibition of all three strains. There was no significant difference in the activity when the strains were exposed to any of the concentrations of $\mathrm{H}_{2} \mathrm{O}_{2}$ tested. The reason for Tet to result in a significant difference in activity compared to $\mathrm{H}_{2} \mathrm{O}_{2}$ may be the differences in size and diffusion ability of the two compounds, as $\mathrm{H}_{2} \mathrm{O}_{2}$ is smaller and may move more freely in a biofilm. Neither of the compounds is excluded by the multidrug efflux pump encoded by pOLA52 (Hansen et al., 2007), but to ensure that the lack of the pump did not affect the observed results, E. coli Genehogs/pOLA52-bla:: $\operatorname{Kan}^{\mathrm{R}}$ was included in the study.

These results indicate that the biofilm mode of growth protects bacteria from some antibacterial compounds, and strains capable of biofilm formation may therefore obtain a higher fitness under some conditions than those incapable of biofilm formation. Thus, the consequences of conjugative transfer of pOLA52 to potentially pathogenic bacteria may be severe, as they may cause hard-to-cure infections on various surfaces.

\section{Expression of the mrk operon}

RT-PCR analysis of the putative mrk operon was performed to determine whether the mrk genes were transcribed as a single mRNA and thereby likely to be organized as an operon. As shown in Fig. 4, we obtained PCR products when using $\mathrm{H} 1$ as template in four RTPCRs, pair-wise linking the $m r k$ genes and indicating organization as an operon, regulated and transcribed by a single promoter. Using a promoter prediction server (www.fruitfly.org/seq_tools/promoter.html), several putative promoters were found to be situated in an IS1 insertion sequence upstream from the operon, which might be involved in the transcription of the mrkABCDF genes.

In K. pneumoniae, the mrk operon encodes type 3 fimbriae, which have previously been detected by immunoblotting with rabbit antiserum against the major subunit MrkA (Di Martino et al., 2003). This was used in this study to verify the expression of type 3 fimbriae on the surface of E. coli Genehogs. As expected, both E. coli Genehogs/pOLA52oqxB:: $\operatorname{Kan}^{\mathrm{R}}(\mathrm{H} 1)$ and K. pneumoniae DSA712 showed

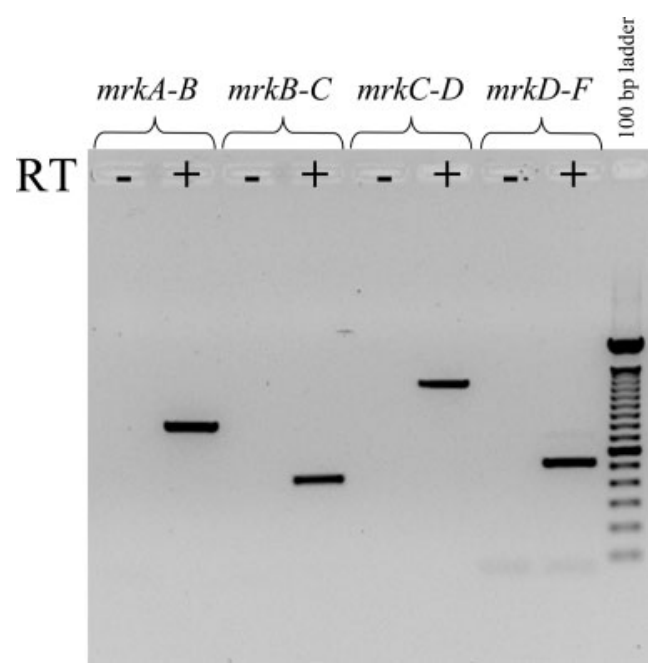

Fig. 4. RT-PCR analysis of $m r k A-F$ transcription in $E$. coli Genehogs/pOLA52-oqxB:: $\operatorname{Kan}^{\mathrm{R}}(\mathrm{H} 1)$; PCR amplification products of either RNA (-) as a negative control or reversetranscribed (RT) DNA (+) were visualized in a $1.2 \%$ agarose gel. $m r k A-B$, RT product generated with mrkBrev and amplified with PCR primers mrkBrevnst1 and mrkAfw; mrkB-C, RT product generated with mrkCrev and amplified with PCR primers mrkCrevnst1 and mrkBfw; mrkC- $D$, RT product generated with mrkFrev1 and amplified with PCR primers mrkDrev and mrkCfw; $m r k D-F$, RT product generated with mrkFrev1 and amplified with PCR primers mrkFrev2 and mrkDfw. 
positive reactions to the anti-MrkA antibody, and E. coli Genehogs did not (data not shown). The results of the RTPCR analysis and the immunoblotting strongly suggest that the mrk operon, contained in pOLA52, encodes type 3 fimbriae, which are expressed by E. coli Genehogs and promote biofilm formation.

\section{Effect of type 3 fimbriae on conjugation frequency of $E$. coli/pOLA52}

The frequency of conjugative transfer events, calculated as transconjugants per donor, was reduced approximately 3750 -fold for the pOLA52- $m r k C:: \mathrm{Kan}^{\mathrm{R}}$ plasmid derivative (transfer frequency $2.4 \times 10^{-5}$ ) compared to the pOLA52oq $\times B:: \mathrm{Kan}^{\mathrm{R}}$ plasmid derivative (transfer frequency $9.0 \times 10^{-2}$ ). This reduction was statistically significant (Student's $t$-test, $P<0.01$ ). Thus, by encoding fimbriae, pOLA52 increases the frequency of its own conjugational transfer, and thereby the plasmid promotes its spread into other bacterial populations. This may enhance the possibility of pOLA52 transfer to potential pathogenic bacteria, which will then acquire the ability to attach to surfaces and become more resistant to antimicrobial agents.

The enhanced conjugation frequency may indicate that, as in $K$. pneumoniae, the type 3 fimbriae, encoded by pOLA52, mediate attachment to abiotic as well as biotic surfaces, the biotic here being intraspecific cell-cell attachment leading to a higher conjugation rate. The ability of type 3 fimbriae to enhance conjugation frequency has not to our knowledge been previously described; however, Dudley et al. (2006) recently reported a similar observation for plasmid-encoded type 4 pili in E. coli. These pili were different from the thicker conjugation pili, but their presence resulted in higher conjugation rates, and enhanced attachment to abiotic (polystyrene and glass coverslips) and to biotic (human epithelial cells) surfaces. Another possible explanation for the observed increased conjugation frequency could be that the type 3 fimbriae (or component(s) thereof) somehow stabilize or aid the conjugation apparatus of the plasmid in the liquid broth. This would require interactions between proteins of the two systems or that the general structural change in the membrane due to the type 3 fimbriae stabilizes the conjugation pili. The reason for higher conjugation frequency of cells expressing the type 3 fimbriae remains to be investigated.

\section{The roles of MrkA and MrkD in biofilm formation}

In the seven clones deficient in biofilm formation, the $\mathrm{Kan}^{\mathrm{R}}$ insertion was localized to the putative mrk operon, specifically in the $m r k A, m r k C$ or $m r k D$ genes. The MrkD adhesin encoded by $m r k D$ has previously been reported to mediate K. pneumoniae biofilm formation on extracellular matrix- and collagen-coated surfaces (Jagnow \& Clegg, 2003), whereas biofilm formation on abiotic plastic surfaces (microtitre plates) was facilitated by the structural fimbrial component MrkA, but independent of MrkD (Langstraat et al., 2001). In contrast to this, our results indicate important roles of both the $m r k A$ and $m r k D$ gene products in biofilm formation in polystyrene microtitre plate wells, as transposon insertion in either gene was shown to abolish biofilm formation. Here we show that the insertions in three of the knockout clones are positioned in the $m r k D$ gene. The $m r k D$ mutants could also abolish the expression of $m r k F$, but previous studies have shown that the absence of MrkF still allows expression of fimbriae. This is indicative of the roles of MrkA, MrkB and MrkC as proteins necessary for the presentation and positioning of the MrkD adhesin in the outer membrane and of the MrkD protein as responsible for the actual adhesion. Further complementation studies are needed to verify this hypothesis. It is possible that the discrepancy is due to the expression of the mrk genes in E. coli in this study, whereas the other studies were performed in K. pneumoniae, or to the fact that we are here studying homologues of the MrkA and MrkD proteins.

\section{Conjugative transfer of pOLA52 to other members of the Enterobacteriaceae, and induction of biofilm formation by these strains}

Type 3 fimbriae are often produced by potentially pathogenic members of the Enterobacteriaceae (Adegbola \& Old, 1983; Allen et al., 1991; Old \& Adegbola, 1985). We successfully transferred pOLA52 to four such members: S. Typhimurium, K. pneumoniae, Kluyvera sp. and

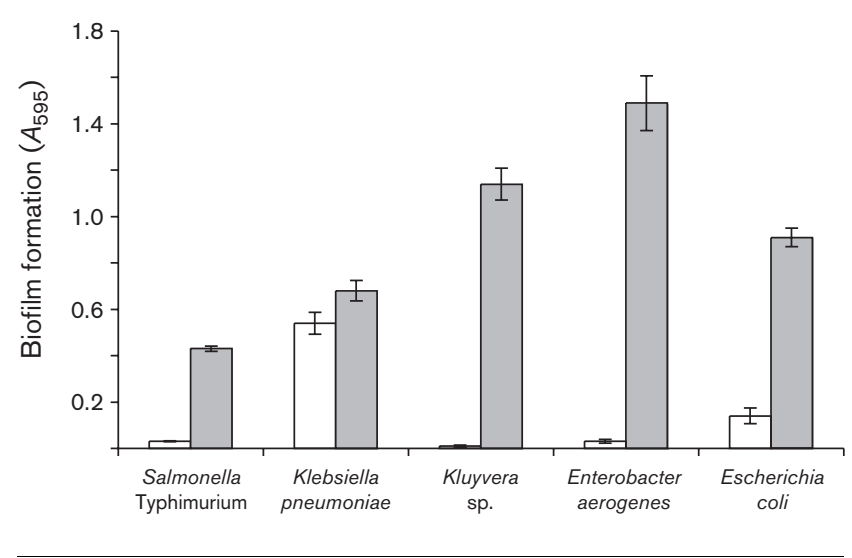

Fig. 5. Biofilm formation of members of the family Enterobacteriaceae: Salmonella Typhimurium DT27, Klebsiella pneumoniae DSA712, Kluyvera sp. MB101 and Enterobacter aerogenes DSM30053, with (grey bars) and without (white bars) pOLA-bla:: $\mathrm{Kan}^{\mathrm{R}}$. Escherichia coli CSH26 \pm pOLA52 was used as control. The cells were incubated in microtitre plate wells for $24 \mathrm{~h}$, followed by quantification of biofilm formation by staining of attached cells and matrix with $\mathrm{CV}$ and spectrophotometric absorbance measurements $\left(A_{595}\right)$. Columns represent means \pm SD for four replicates. 
Enterobacter aerogenes. As shown in Fig. 5, statistically significant increases in biofilm formation were observed in all strains harbouring plasmid pOLA52-bla:: $\operatorname{Kan}^{\mathrm{R}}(6.5-$ 114 -fold induction; Student's $t$-test, $P<0.01)$. Although still statistically significant, the increase in biofilm formation was less pronounced in $K$. pneumoniae when compared to the remaining strains, most likely because this strain contains the mrk operon on the chromosome, and therefore expresses type 3 fimbriae regardless of harbouring pOLA52. These results are strongly indicative of pOLA52-encoded type 3 fimbriae expression of these Enterobacteriaceae strains, enhancing their attachment and biofilm formation, which may lead to increased virulence. It remains to be investigated whether the type 3 fimbriae, expressed from pOLA52, constitute any advantage in adherence to animal cells. We will test this, using model animal systems, in the near future.

\section{Concluding remarks}

The results of this and previous studies reveal some disturbing characteristics of pOLA52, regarding human health. The plasmid encodes a multidrug efflux pump of the RND family, capable of exporting a wide range of antibiotics, detergents and disinfectants (Hansen et al., 2004, 2007). Furthermore, the bla gene of the plasmid encodes $\beta$-lactamase, conferring resistance to $\beta$-lactam antibiotics (Hansen et al., 2004). The plasmid also encodes type 3 fimbriae, promoting attachment to abiotic and biotic surfaces, biofilm formation and its own spread by increased conjugative transfer rates. To our knowledge, this is the first example of adhesive fimbriae and a multidrug efflux pump being encoded on a single, conjugative plasmid. The ability to form biofilm and export a variety of chemicals will, in many environments, increase the fitness of the host cell, as demonstrated in this study with exposure to Tet. These traits are therefore likely to contribute to selectively stabilizing the presence of pOLA52 in its present hosts, but also to spread of the plasmid to new hosts. As demonstrated here, pOLA52 can easily be transferred from $E$. coli to other, potentially pathogenic members of the Enterobacteriaceae, which may increase the virulence of these recipient strains. Infections caused by a pathogenic bacterial strain that has acquired pOLA52 may therefore be difficult to cure. Previously we have found several $E$. coli isolates from pigs harbouring the oq $x A B$ genes on plasmids (Hansen et al., 2005). It remains to be investigated if these plasmids also encode the ability to express type 3 fimbriae. The fact that pOLA52 originates from swine manure renders this scenario worryingly realistic.

\section{ACKNOWLEDGEMENTS}

We thank Arlette Darfeuille-Michaud for kindly providing the MrkA polyclonal antibody. We also thank Karin Pinholdt Vestberg for excellent technical assistance. The study was partly funded by a grant from the Danish Natural Science Research Council, ref. 272-05-0325.

\section{REFERENCES}

Adegbola, R. A. \& Old, D. C. (1983). Fimbrial haemagglutinins in Enterobacter species. J Gen Microbiol 129, 2175-2180.

Allen, B. L., Gerlach, G. F. \& Clegg, S. (1991). Nucleotide sequence and functions of $m r k$ determinants necessary for expression of type 3 fimbriae in Klebsiella pneumoniae. J Bacteriol 173, 916-920.

Brunder, W., Khan, A. S., Hacker, J. \& Karch, H. (2001). Novel type of fimbriae encoded by the large plasmid of sorbitol-fermenting enterohemorrhagic Escherichia coli $\mathrm{O} 157: \mathrm{H}(-)$. Infect Immun 69, 4447-4457.

Burmølle, M., Hansen, L. H. \& Sørensen, S. J. (2005). Use of a wholecell biosensor and flow cytometry to detect AHL production by an indigenous soil community during decomposition of litter. Microb Ecol 50, 221-229.

Burmølle, M., Webb, J. S., Rao, D., Hansen, L. H., Sørensen, S. J. \& Kjelleberg, S. (2006). Enhanced biofilm formation and increased resistance towards antimicrobial agents and bacterial invasion are caused by synergistic interactions in multispecies biofilms. Appl Environ Microbiol 72, 3916-3923.

Castonguay, M. H., van der Schaaf, S., Koester, W., Krooneman, J., van der Meer, W., Harmsen, H. \& Landini, P. (2006). Biofilm formation by Escherichia coli is stimulated by synergistic interactions and co-adhesion mechanisms with adherence-proficient bacteria. Res Microbiol 157, 471-478.

de Lipthay, J. R., Barkay, T. \& Sørensen, S. J. (2001). Enhanced degradation of phenoxyacetic acid in soil by horizontal transfer of the $t f d A$ gene encoding a 2,4-dichlorophenoxyacetic acid dioxygenase. FEMS Microbiol Ecol 35, 75-84.

Di Martino, P., Cafferini, N., Joly, B. \& Darfeuille-Michaud, A. (2003). Klebsiella pneumoniae type 3 pili facilitate adherence and biofilm formation on abiotic surfaces. Res Microbiol 154, 9-16.

Donlan, R. M. \& Costerton, J. W. (2002). Biofilms: survival mechanisms of clinically relevant microorganisms. Clin Microbiol Rev 15, 167-193.

Dudley, E. G., Abe, C., Ghigo, J. M., Latour-Lambert, P., Hormazabal, J. C. \& Nataro, J. P. (2006). An IncI1 plasmid contributes to the adherence of the atypical enteroaggregative Escherichia coli strain C1096 to cultured cells and abiotic surfaces. Infect Immun 74, 2102-2114.

Fux, C. A., Costerton, J. W., Stewart, P. S. \& Stoodley, P. (2005). Survival strategies of infectious biofilms. Trends Microbiol 13, 34-40.

Gerlach, G. F., Allen, B. L. \& Clegg, S. (1988). Molecular characterization of the type $3(\mathrm{MR} / \mathrm{K})$ fimbriae of Klebsiella pneumoniae. J Bacteriol 170, 3547-3553.

Ghigo, J. M. (2001). Natural conjugative plasmids induce bacterial biofilm development. Nature 412, 442-445.

Hansen, L. H., Sørensen, S. J. \& Jensen, L. B. (1997). Chromosomal insertion of the entire Escherichia coli lactose operon, into two strains of Pseudomonas, using a modified mini-Tn5 delivery system. Gene 186, 167-173.

Hansen, L. H., Johannesen, E., Burmølle, M., Sørensen, A. H. \& Sørensen, S. J. (2004). Plasmid-encoded multidrug efflux pump conferring resistance to olaquindox in Escherichia coli. Antimicrob Agents Chemother 48, 3332-3337.

Hansen, L. H., Sørensen, S. J., Jørgensen, H. S. \& Jensen, L. B. (2005). The prevalence of the OqxAB multidrug efflux pump amongst olaquindox-resistant Escherichia coli in pigs. Microb Drug Resist 11, 378-382.

Hansen, L. H., Jensen, L. B., Sørensen, H. I. \& Sørensen, S. J. (2007). Substrate specificity of the OqxAB multidrug resistance pump in 
Escherichia coli and selected enteric bacteria. J Antimicrob Chemother 60, 145-147.

Hornick, D. B., Allen, B. L., Horn, M. A. \& Clegg, S. (1991). Fimbrial types among respiratory isolates belonging to the family Enterobacteriaceae. J Clin Microbiol 29, 1795-1800.

Jagnow, J. \& Clegg, S. (2003). Klebsiella pneumoniae MrkD-mediated biofilm formation on extracellular matrix- and collagen-coated surfaces. Microbiology 149, 2397-2405.

Jefferson, K. K. (2004). What drives bacteria to produce a biofilm? FEMS Microbiol Lett 236, 163-173.

Klausen, M., Gjermansen, M., Kreft, J. U. \& Tolker-Nielsen, T. (2006). Dynamics of development and dispersal in sessile microbial communities: examples from Pseudomonas aeruginosa and Pseudomonas putida model biofilms. FEMS Microbiol Lett 261, 1-11.

Langstraat, J., Bohse, M. \& Clegg, S. (2001). Type 3 fimbrial shaft (MrkA) of Klebsiella pneumoniae, but not the fimbrial adhesin (MrkD), facilitates biofilm formation. Infect Immun 69, 5805-5812.

Levine, M. M. (1987). Escherichia coli that cause diarrhea: enterotoxigenic, enteropathogenic, enteroinvasive, enterohemorrhagic, and enteroadherent. J Infect Dis 155, 377-389.

Livrelli, V., De Champs, C., Di Martino, P., Darfeuille-Michaud, A., Forestier, C. \& Joly, B. (1996). Adhesive properties and antibiotic resistance of Klebsiella, Enterobacter, and Serratia clinical isolates involved in nosocomial infections. J Clin Microbiol 34, 1963-1969.

Miller, J. H. (1972). Experiments in Molecular Genetics. Cold Spring Harbor, NY: Cold Spring Harbor Laboratory.

O'Toole, G. A. \& Kolter, R. (1998). Initiation of biofilm formation in Pseudomonas fluorescens WCS365 proceeds via multiple, convergent signalling pathways: a genetic analysis. Mol Microbiol 28, 449-461.

Old, D. C. \& Adegbola, R. A. (1985). Antigenic relationships among type-3 fimbriae of Enterobacteriaceae revealed by immunoelectronmicroscopy. J Med Microbiol 20, 113-121.

Olsen, J. E., Brown, D. J., Thomsen, L. E., Platt, D. J. \& Chadfield, M. S. (2004). Differences in the carriage and the ability to utilize the serotype associated virulence plasmid in strains of Salmonella enterica serotype Typhimurium investigated by use of a self-transferable virulence plasmid, pOG669. Microb Pathog 36, 337-347.

Reisner, A., Haagensen, J. A., Schembri, M. A., Zechner, E. L. \& Molin, S. (2003). Development and maturation of Escherichia coli K12 biofilms. Mol Microbiol 48, 933-946.

Reisner, A., Holler, B. M., Molin, S. \& Zechner, E. L. (2006). Synergistic effects in mixed Escherichia coli biofilms: conjugative plasmid transfer drives biofilm expansion. J Bacteriol 188, 3582-3588.

Sambrook, J., Fritsch, E. F. \& Maniatis, T. (1989). Molecular Cloning: a Laboratory Manual, 2nd edn. Cold Spring Harbor, NY: Cold Spring Harbor Laboratory.

Sauer, F. G., Barnhart, M., Choudhury, D., Knight, S. D., Waksman, G. \& Hultgren, S. J. (2000). Chaperone-assisted pilus assembly and bacterial attachment. Curr Opin Struct Biol 10, 548-556.

Schurtz, T. A., Hornick, D. B., Korhonen, T. K. \& Clegg, S. (1994). The type 3 fimbrial adhesin gene $(m r k D)$ of Klebsiella species is not conserved among all fimbriate strains. Infect Immun 62, 4186-4191.

Silhavy, T. J., Berman, M. L. \& Enquist, L. W. (1984). Experiments with Gene Fusion. Cold Spring Harbor, NY: Cold Spring Harbor Laboratory.

Sørensen, A. H., Hansen, L. H., Johannesen, E. \& Sørensen, S. J. (2003). Conjugative plasmid conferring resistance to olaquindox. Antimicrob Agents Chemother 47, 798-799.

Sutherland, I. (2001). Biofilm exopolysaccharides: a strong and sticky framework. Microbiology 147, 3-9.

Tarkkanen, A. M., Virkola, R., Clegg, S. \& Korhonen, T. K. (1997). Binding of the type 3 fimbriae of Klebsiella pneumoniae to human endothelial and urinary bladder cells. Infect Immun 65, 1546-1549.

Vila, J., Vargas, M., Henderson, I. R., Gascon, J. \& Nataro, J. P. (2000). Enteroaggregative Escherichia coli virulence factors in traveler's diarrhea strains. J Infect Dis 182, 1780-1783.

Williams, P. \& Tomas, J. M. (1990). The pathogenicity of Klebsiella pneumoniae. Rev Med Microbiol 1, 196-204.

Edited by: J. G. Shaw 